\title{
Association of Aquaculture Environment Microbiota with Metabolism in American Shad (Alosa Sapidissima)
}

Jia Du ( $\square$ djhd@hdu.edu.cn )

Hangzhou Dianzi University https://orcid.org/0000-0003-3638-5646

Qinghua Liu

Hongze Fishseeds Bio-technology

Keqin Zhang

Soochow University

Chengxiang Zhang

Jiangyin Aquaculture Technology Station

Gaohua Yao

Zhejiang Provincial Aquaculture Technology Promotion Station

\section{Research Article}

Keywords: Microbiota, Metabolic disruption, Aquaculture environment, Transcriptomic, American shad

Posted Date: August 4th, 2021

DOI: https://doi.org/10.21203/rs.3.rs-719548/v1

License: @ (i) This work is licensed under a Creative Commons Attribution 4.0 International License. Read Full License 


\section{Abstract}

The environment microbiome affects the growth and development of fish species. Information of the environment microbiome is beneficial to increase the production of fish in different aquaculture systems. In this study we analyzed differences in environmental microbial composition, intestinal metabolites and differentially expressed genes (DEGs) in American shad living in tank aquaculture and pond aquaculture environment. The results demonstrated that the dominant phyla were Bacteroidetes, Actinobacteria, Fusobacteria, Gemmatimonadetes, Firmicutes, Acidobacteria, Nitrospirae and Epsilonbacteraeota in two different environments. There were significantly changed of metabolites in different aquaculture environment, including DP-ethanolamine, Lproline, sulfuric acid, L-valine, L-tryptophan, creatinine, uric acid and L-isoleucine. The transcriptome data revealed eight genes (As23G026314, As04G005148, As21G024434, As04G005193, As23G026314, As13G016035, As02G001872 and As07G009244) related to metabolisms significantly changed in pond aquaculture group compared to tank aquaculture group. In addition, the body weight, amino acid metabolism, and glycerophospholipid metabolism of American shad also significantly changed in the pond aquaculture environment. Therefore, identifying the predominant microbiome in the aquaculture environment may be prevent the disease from occurring and maintain healthy fish reared in the aquaculture environment.

\section{Introduction}

Microorganisms play the critical role in the sediment and water of various aquaculture environments due to participating in biogeochemical cycles and food network interactions (Wörmer et al., 2019). Bacteria and some kinds of eukaryotic microorganisms involve in maintain a balance of elements (such as nitrogen, phosphorus, sulfur) and participate in a range of physiological functions in the aquatic environment and sediment (Grujcic et al., 2018). The nutrients of aquatic organisms usually sink into the sediment of aquaculture water and eventually become a source of nutrients for bacterial and eukaryotic (Shaughnessy et al., 2019). In addition, microorganisms affect the quality of water, such as involved in aerobic chemoheterotrophy and phototrophy, organic matter decomposition, and sulfur compound transformation, which indirectly affected the growth of aquatic organisms (Kumar et al., 2019; Mahmoudi et al., 2017; Ul-Hasan et al., 2019). Transcriptomics and metabolomics are often used to analyze the specific signaling pathways associated with environment, physiology and health (Beale et al., 2016). The transcriptomics and metabolomics could reveal the critical signaling pathways and metabolism regulation networks, which affecting the aquatic organism's carbohydrate metabolism and growth (Su et al. 2021; Qiu et al., 2020; Zhou et al., 2017). To understand the mechanism of environmental influence on the growth of aquatic organisms, the related transcriptomics and metabolomics have integrated into the same study.

American shad (Alosa sapidissima) is an anadromous species and mainly originates in the western Atlantic Ocean and the east coast of Canada and the United States (Nack et al., 2019). To alternative the Reeves shad (Tenualosa reevesii), artificial farming techniques of American shad are becoming mature in China (Liu et al., 2021). However, American shad is vulnerable and overreacting to the environment, and any improper handling will result in the death of this species. Some researchers have focused on enhancing the reproductive capacity and improving the breeding conditions of American shad, including artificially induced spawning, environmental condition regulation, and breeding improved variety (St. Pierre, 1992; Liu et al., 2006). The breeding conditions, such as water quality, temperature, illumination intensity, and oxygen content, can induce oxidative stress reaction, enhance mortality, and affect the growth and development of American shad. It has suggested that the environmental requirements of its ontogenetic development are vital for the growth performance of American shad broodstocks (Liu et al., 2021).

Pond aquaculture plays an important role in the freshwater aquaculture of China, and the application area nearly accounts for $50 \%$ of the country's aquaculture (National Bureau of Fisheries and China Society of Fisheries, 2019). However, commercial aquaculture models have also emerged in China, especially in American shad aquaculture (Liu et al., 2021). The microbial species in the water and sediment are closely relevant to the fish culture environment, because of the activities of the aquaculture species and the characteristics of environmental requirements in aquaculture, including dissolved oxygen, lighting, and the frequency of mechanical stirring. Microorganisms in commercial aquaculture and pond aquaculture are also closely related to the culture environment of fish (Zhao et al., 2015). The growth and development of American shad were different in tank aquaculture and pond aquaculture. The correlation between the development of American shad and microorganisms has seldom been analyzed in the aquaculture environment. In addition, the characteristics and differences of microorganisms' distributions in tank and pond aquaculture are still unclear. Thus, in the survey, we compared microorganisms in two different aquaculture tankage in Hongze of 
China and analyzed their influence on the growth and development, intestinal metabolites, and differentially expressed genes (DEGs) in the gut of American shad. The purpose is to reveal the differences in the distribution patterns of microorganism species between tank aquaculture and pond aquaculture and show the influence on growth, metabolites, and transcription of crucial genes in different aquaculture environments. This study provides the information to evaluate growth parameters in the different aquatic pond, which is helpful to improve the efficiency of pond aquaculture.

\section{Materials And Methods}

\section{Chemicals and reagents}

Water, methanol, acetonitrile, and formic acid were purchased from CNW Technologies GmbH (Düsseldorf, Germany). L-2chlorophenylalanine was purchased from Shanghai Hengchuang Biotechnology Co., Ltd. (Shanghai, China). DNeasy PowerSoil Kit (Cat. No. 12888) and QIAamp 96 PowerFecal QIAcube HT Kit (Cat. No. 51531) were purchased from QIAGEN (Germany). Qubit dsDNA Assay Kit (Cat. No. Q32854) was purchased from Life Technologies (America). Tks Gflex DNA Polymerase (Cat. No. R060B) was purchased from Takara (Japan).

\section{Fish culture conditions, treatments and sample collection}

Two-month-old fish were raised in a greenhouse tank recirculating aquaculture system (RAS) and underwent sixth-generation selection in indoor concrete tanks at Hongze Fishseeds Biotech. Inc., Jiangsu, China. RAS can ensure the excellence of the water quality due to the physical filtration, oxygenation and bio-filtration (Liu et al., 2021). The system was a specially designed dualdrain RAS equipped with an online infrared dome camera to monitor the growth, maturation and natural spawning behavior of shad broodstocks. Other two-month-old fish were also raised in a greenhouse pond aquaculture system at Hongze Fishseeds Biotech. Inc., Jiangsu, China. Temperature and dissolved oxygen were measured by a YSI 550A (Y.S.I. Environmental Inc.) every morning in both locations. Water quality was monitored by testing ammonia and nitrite levels every four days in both locations. Both recirculating aquaculture system and the pond were maintained under a natural photoperiod in the greenhouse, with the sunlight intensity attenuated by a roof cover with $40 \%$ light penetration. The daily average light intensity ranged from 800 to 1600 lux. Fish were fed commercial pellets three times a day for 20 min each time during the entire culture period.

Sixty fish (two months postfertilization) were randomly selected and divided into three tanks or three ponds (20 fish per tank or pond). After thirteen month cultured, the ten-individual fish from each tank and pond $(n=3)$ sampled to measure the body weight. Then these fish were washed with purified water and euthanized. The intestinal parts were dissected and gathered for metabolites and RNA. Four biological replicates per group were used for LC-MS analysis and three biological replicates per group were used for RNA-Seq analysis. The sediment at the bottom of the tank aquaculture and pond aquaculture was also collected. Four tubes ( $>2 \mathrm{mg} /$ tube) of sediment were gathered for each group to ensure a sufficient number of DNA.

\section{Sample preparation and analysis}

\section{Metabolite extraction}

The process of metabolite extraction referenced Lu et al., (2016). The ACQUITY UHPLC system (Waters Corporation, Milford, USA) coupled to an AB SCIEX Triple TOF 5600 System (AB SCIEX, Framingham, MA) was used to reveal the metabolic profiles with ESI. The modes of positive and negative ion executed by using the $\mathrm{C} 18 \mathrm{column}$. Water/formic acid and acetonitrile/formic acid were used to elute, and the separation parameter followed the instructions.

\section{RNA extraction, library construction and sequencing}

RNA was extracted from $3 \mathrm{mg}$ of intestinal tissue using a mirVana miRNA Isolation Kit (Ambion). The RNA-Seq process was performed as described in Zhang et al. (2021). RNA integrity was evaluated using an Agilent 2100 Bioanalyzer (Agilent Technologies, Santa Clara, CA, USA). Samples with an RNA integrity number (RIN) $\geq 7$ were used for subsequent analysis. Libraries were constructed using a TruSeq Stranded mRNA LT Sample Prep Kit (Illumina, San Diego, CA, USA) according to the manufacturer's instructions. Then, these libraries were sequenced on an Illumina sequencing platform (Illumina HiSeq X Ten), and $125 \mathrm{bp} / 150$ bp paired-end reads were generated. 


\section{DNA extraction and library construction}

DNA was extracted from gut samples using TruSeq Nano DNA LT Sample Prepararion Kit (Illumina). The V4 region of bacterial 16S rRNA was amplified using DNA samples. Agencourt AMPure XP (BECKMAN COULTER, USA) was used for purifying PCR products. Sequencing libraries were constructed using KAPA Library Quantification Kits (Illumina, USA). The Illumina Miseq platform was used for paired-end sequencing (Kapa Biosystems, USA).

\section{Data preprocessing and statistical analysis}

\section{LC-MS metabolomics data analysis}

LC-MS metabolomic analysis was done by OE Biotech Co. Ltd. (Shanghai, China). Progenesis QI software was used for acquired LC-MS raw data analysis (Waters Corporation, Milford, USA). Progenesis QI Data Processing Software was used to analyze the metabolites (Waters Corporation, Milford, USA). The public databases were referenced to explore, including http://www.hmdb.ca/; http://www.lipidmaps.org/ and in-house-developed databases. The changes of metabolite were analyzed by Principal component analysis (PCA) and orthogonal partial least-squares-discriminant analysis (OPLS-DA). The Hotelling's T2 region shows the $95 \%$ confidence interval in the model. Variable importance in the projection (VIP) shows the contribution rate of each variable in the OPLS-DA model. The differential metabolites were obtained based on the combination of a statistically significant threshold of variable influence on projection (VIP) values selected from the OPLS-DA model and $p$ values from a two-tailed Student's t-test on the normalized peak areas. The differential metabolites were with $\mathrm{VIP}>1$ and $p$ values $<0.05$.

\section{RNA sequencing data analysis}

Transcriptome sequencing and analysis were also done by OE Biotech Co., Ltd. Trimmomatic was used to process raw data (raw reads) (Bolger et al., 2014). Clean reads were obtained by removing poly-N sequences and low-quality reads. Hisat2 was used to map the clean reads and genome (Kim et al., 2015). For transcript-level quantification, bowtie2 was used to analyze the values of the transcript. DESeq (2012) functions were used to identify the DEGs based on SizeFactors and nbinom Test. The significantly differential expression was showed with the thresholds of $P<0.05$ and fold change $>2$ or fold change $<0.5$. The transcript expression patterns were executed by hierarchical cluster analysis of DEGs. DEGs were analyzed by GO term enrichment and KEGG pathway enrichment following the hypergeometric distribution (Kanehisa et al., 2008).

\section{S rRNA metagenetics and bioinformatics analysis}

FASTQ format was used for the raw sequence data. Trimmomatic software (Bolger et al., 2014) was used to preprocess the pairedend reads and eliminated ambiguous bases (N). FLASH software was used to assemble the left paired-end reads (Reyon et al., 2012) as follows: overlapping was from $10 \mathrm{bp}$ to $200 \mathrm{bp}$, and the mismatch rate was from $0 \%$ to $20 \%$. QIIME software was used to denoise and clean the reads (Caporaso et al., 2010) (version 1.8.0). The primer sequences were removed from the clean reads, and Vsearch software was used to execute the OTUs (Rognes et al., 2016). QIIME package was used to select the representative read in every OTU. RDP classifier was used to annotate and blast the usual reads (Wang et al., 2007). All representative reads were annotated and blasted against Unite database (ITSs rDNA) using blast (Lobo et al., 2008).

\section{Functional analysis of integrated metabolomic and transcriptomic data}

KEGG database (https://www.kegg.jp/) was used to define the metabolic pathways and integrated metabolomic and transcriptomic datasets. The markers list was introduced into the KEGG database to find out metabolic pathways induced by ENR treatment. The specific metabolite is related to a particular gene if they shared one common KEGG pathway.

\section{Results}

\section{Bodyweight}

As shown in Fig. 1, the body weight $(577.11 \pm 21.56 \mathrm{~g})$ was significantly increased in the pond aquaculture groups compared to that in the tank aquaculture group $(559.63 \pm 20.19 \mathrm{~g})$.

\section{Metabolomic Alterations Induced By Different Aquaculture Environments}


Metabolomic analysis was done to analyze the alterations in metabolic profiles of fish gut cultured in tank aquaculture and pond aquaculture. Ninety-four metabolites significantly changed $(p<0.05)$ after culture in the pond aquaculture $(72$ metabolites upregulated and 22 metabolites downregulated) (Supplementary Fig. 1). Several of the metabolites were associated with the tank aquaculture and the pond aquaculture (Supplementary Fig. 1). The heatmap shows the relative changes in the metabolite concentrations of each group with colors (Fig. 2A). Red and blue represent the upregulation and downregulation of metabolites, respectively. The OPLS-DA score plot shows a clear separation of metabolic profiles between the tank aquaculture group and the pond aquaculture group (Fig. 2B). In the OPLS-DA model, $R_{2} X=0.787, R_{2} Y=0.93, Q 2$ (cum) $=0.441$ and $R 2=0.943$, indicating the usable of the model. The VIP values and coefficients of the OPLS-DA model were analyzed to find out the effect of metabolites on metabolic alterations in different aquaculture environments (Supplementary material 1). The most metabolites affected in pond aquaculture were related to aminoacyl-tRNA biosynthesis, phenylalanine, tyrosine and tryptophan biosynthesis, $A B C$ transporters and valine, leucine and isoleucine biosynthesis metabolic pathways. Metabolic pathways of top- 20 were shown in Fig. $2 \mathrm{C}$. Four metabolic pathways were significantly different at $P>0.01$, while seven metabolic pathways were significantly different at $P>0.05$. The coefficients and p-value of the metabolic pathways were analyzed to show the correlation of metabolites on metabolomic alterations in the different aquaculture environments (Supplementary material 2). The significantly enriched pathway was selected to construct a bubble diagram. Figure 2D showed that among the altered metabolic pathways, the rich factor was the largest for the aminoacyl - tRNA biosynthesis and ABC transporters pathways. And these two pathways were related to three common metabolites such as L-Histidine, L-Lysine and L-Valine.

\section{Transcriptomics Of Different Cultured Environments}

276 DEGs were found in the tank aquaculture and the pong aquaculture treatment groups $(P<0.05)$, precisely, 169 upregulated and 107 downregulated. Two parts of hierarchical clusters were shown in different cultured environments according to DEGs expression levels (Fig. 3A, cluster A and cluster B). Cluster A represented upregulated genes, while cluster B represented downregulated genes. As shown in Table 1, the top-twenty of DEGs involved in different molecular functions, such as protein binding, succinate dehydrogenase activity, iron ion binding, inorganic anion exchanger activity and motor activity. The volcano plot revealed the overall distribution of the DEGs (Supplementary Fig. 2). The distribution comparison diagram of DEGs and all genes by GO enrichment analysis at level 2 was shown in Fig. 3B. The three major categories, such as biological processes, cellular components and molecular functions, were revealed in Fig. 3B. The distribution comparison diagram of upregulated and downregulated DEGs were shown in GO level 2 analysis (Supplementary Fig. 3). Among the identified pathways, the thirty most important pathways involved in lipid metabolism and energy metabolism were showed in Fig. 3C, and some DEGs involved in growth and development. KEGG database showed the diverse ranges of functionally characterize DEGs, with the 276 DEGs between the tank aquaculture and the pond aquaculture groups assigned to 168 pathways. The top-twenty pathways identified by KEGG enrichment analysis were the focus. A bubble diagram was constructed using the -log10 ( $\mathrm{p}$-value) to visualize the expression patterns of DEGs. The pathways of DEGs were mainly included viral myocarditis, immunodeficiency, allograft rejection, and autoimmune thyroid disease pathways (Fig. 3D).

\section{Association between the altered pathways with metabolites and transcriptomic pathway}

The KEGG database were used to analyze the effects of two environment on transcriptomics pathway and metabolomics pathway. The pond aquaculture groups induced significant alterations in the metabolites and genes in the gut of American shad compared to the tank aquaculture groups. To investigate the potential associations, Venn diagrams (Fig. 4A) were generated by identifying the common KEGG pathways. Five of the common metabolite pathways (glycerophospholipid metabolism, arginine and proline metabolism, purine metabolism, valine, leucine and isoleucine degradation, and glycine, serine and threonine metabolism) shared nine genes (As13G016035, As21G024434, As04G005193, As04G005148, As02G001872, As07G009244, As15G018635, As13G016748, and As23G026314) and eight metabolites (CDP-ethanolamine, L-proline, sulfuric acid, L-valine, L-tryptophan, creatinine, uric acid and L-isoleucine). The total metabolites levels increased upon pond aquaculture, and the nine genes expression changed (Table 2 in detail). The association network map was constructed based on the results of the association analysis of differential genes and differential metabolites (Fig. 4B). The total differential metabolites levels increased upon pond aquaculture, whereas up to 5 genes (As13G016035, As02G001872, As07G009244, As15G018635 and As23G026314) related to the glycine, serine and threonine metabolism, purine metabolism, arginine and proline metabolism, glycerophospholipid metabolism appeared to downregulate (Table 2 in detail, in Fig. 5A-D). In addition, the total differential metabolites levels increased upon pond 
aquaculture, whereas up to 4 genes (As13G016748, As21G024434, As04G005193 and As04G005148) related to valine, leucine and isoleucine degradation and glycerophospholipid metabolism appeared to be upregulated (Table 2 and, in detail, in Fig. 5E). The effect of metabolites in different aquaculture environmental remains to be investigated.

\section{Microbial Community Analysis}

Microbial community analysis according to high-throughput sequencing was done to reveal the changes of microbiome community in the tank and pond aquaculture environment. The two environment samples were all dominated by the phyla of Proteobacteria and Bacteroidetes (Fig. 6A). The compositions of the microbial communities in all the samples were similar at the rank of the phylum. Proteobacteria was the most abundant bacterial phylum in all eight samples, accounting for approximately $60 \%$ of the total species. The number of proteobacteria was more significant in the pond environment compared to the tank environment. The relative changes in the microbial abundance showed with colors in the heatmap (Fig. 6B). We use alpha diversity indices of the Chao index (Fig. 6C), and the Shannon index (Fig. 6D) to confirm the results. This is a significantly higher diversity of the microbiome in the pond environment than the tank environment group. We performed beta diversity index, PCA analysis, PCoA analysis, and UPGMA sample hierarchical cluster analysis to determine the classified bacterial taxa between different groups (Fig. 7). In addition, a biomarker analysis using the linear discriminant analysis (LDA) effect size (LEfSe) method was executed to determine the classified bacterial taxa with significant differences in abundance. As shown in Fig. 8A and B, twenty-eight bacterial clades showed statistically significant changes with an LDA threshold of 4.1. Nine bacteria were significantly enriched in pond aquaculture environment group, while nineteen clades showed an abundance advantage in the tank aquaculture environment group. The relative abundance of phylum was shown in Fig. 8C, bacteroidia, alphaproteobacteria, fusobacteria, firmicutes, gemmatimonadetes, acidobacteria, nitrospirae and epsilonbacteraeota were enriched in pond aquaculture environment samples. The species correlation at various taxonomic levels reflected in the species correlation network map (Fig. 8D).

\section{Discussion}

Our research reveals the differences in the sediment microbial communities in tank aquaculture and pond aquaculture of American shad (Alosa sapidissima). We analyzed the microbiome in the system and provided information on the effect of the environment factor on the American shad growth. The result showed that the microbiome was dominated by the phyla of Proteobacteria, Fusobacteria, Bacteroidetes and Actinobacteria in the pond aquaculture environment. This result is consistent with other findings in the pond environments. For example, Dai et al., (2021) found bacteroidetes, proteobacteria and actinobacteria were more abundant in the pond environment. The pond aquaculture induced significant alterations in the metabolomes and transcriptomic pathways of the gut compared to tank aquaculture. For example, the total amino acid metabolites level increased upon pond aquaculture, whereas up to 5 genes related to the pathway appeared to be downregulated. DP-ethanolamine, L-proline, sulfuric acid, L-valine, Ltryptophan, creatinine, uric acid, and L-isoleucine increased in the pond aquaculture group and positively correlated with the family of B8 (Fusobacteria;f_Fusobacteriaceae) and B9 (Planctomycetes; f_Gemmataceae). Similarly, lysine increased in the ponk aquaculture group and positively correlated with the families of B5 (Chlamydiae;f_unassigned), B14 (Proteobacteria;f_unassigned) and B16 (Proteobacteria; f_Neisseriaceae). Fusobacteriaceae, aeromonadaceae and moraxellaceae were enriched in the pond environment compared to the tank environment. Microorganisms in sediment were mainly related to the enzymes of organic matter decomposition (Dai et al., 2021), which served as a natural nutritional supplement to enhance aquatic organisms' growth (Addo et al., 2021). The nutritional supplement is closely related to the growth development of aquatic animals. The results indicated that greater abundance and diversity of microbial communities in pond aquaculture group induced better growth condition in American shad. The results of the microbial community are consistent with growth indicators.

The metabolomic and transcriptomic analysis showed the metabolic disruption of the gut of American shad. CDP-ethanolamine, Lproline, sulfuric acid, L-valine, L-tryptophan, creatinine, uric acid, and L-isoleucine increased in the pond aquaculture group compared to the tank aquaculture group. The integration data at the pathway level showed the affected metabolic routes in the gut of American shad after the pond aquaculture, including glycerophospholipid metabolism, arginine and proline metabolism, purine metabolism, valine, leucine and isoleucine degradation, and glycine, serine and threonine metabolism. The total energy of fish meets the basic metabolic needs, including growth, development and reproduction (Bunnell et al. 2003; Ye et al., 2011). Amino acids and glycerophospholipid have a crucial role in the development of fish, including providing essential fatty acids, essential 
protein and enough energy for muscle, tissue and gonad development (Masrizal et al., 2015). The results of both transcriptomic and metabolomic are in agreement with growth indicators.

Fish is the source of the essential protein and fatty acids for humans. At present, aquaculture is the main source for fish production and consumption (FAO, 2020). Bjrnevik et al., (2017) revealed that the improper aquaculture pattern affected the quality and the taste of fish flesh, including reducing protein content. Ostbye et al., (2018) reported that the quality of fish, including nutritional composition, was primarily affected by environmental factors. This conclusion is in agreement with our study, indicating the changes of metabolites in a different aquaculture environment, involving DP-ethanolamine, L-proline, sulfuric acid, L-valine, Ltryptophan, creatinine, uric acid, and L-isoleucine. The transcriptome data revealed four genes (As23G026314, As04G005148, As21G024434, and As04G005193) related to these metabolisms (valine, leucine and isoleucine degradation, and glycerophospholipid) significantly up-regulated. In comparison, the four genes (As23G026314, As13G016035, As02G001872 and As07G009244) related to metabolisms (glycine, serine and threonine metabolism, purine metabolism, and arginine) significantly down-regulated in pond aquaculture group. Based on our analysis, numerous genes and metabolites differentially expressed between the tank and pond aquaculture groups, in agreement with earlier investigations. For example, the gene, lipid metabolism and amino acid metabolism are the most direct manifestation of the response of plants changes in the external environment (Sun et al., 2020; Yang et al., 2019). Xu et al., (2016) also reported that glycine, serine, arginine, proline metabolism and threonine metabolism of oriental river prawn Macrobrachium Nipponese were significant differences in response to acute and chronic environment factor stress. This survey provides valuable information on the diversity of microbiomes in different aquaculture. In addition, the potential effects of microbiomes on the growth of these fishes are also mentioned.

\section{Conclusion}

Our research analyzed the differences in the distributions of microbial communities between the tank aquaculture and the pond aquaculture environment. We found that the dominant phyla were Bacteroidetes, Actinobacteria, Fusobacteria, Gemmatimonadetes, Firmicutes, Acidobacteria, Nitrospirae and Epsilonbacteraeota in both aquaculture environment, by comparing microorganisms in different aquaculture ponds from the same location. The bacterial abundance significantly increased in the pond aquaculture than that in tank aquaculture. Some identified differential genes and metabolites were associated with the response of American shad to different aquaculture environments. In conclusion, the body weight, amino acid metabolism, and glycerophospholipid metabolism of American shad significantly changed due to the pond aquaculture environment. Taken together, during popular aquaculture, the interaction effect of the aquaculture environment on cost benefits should be carefully evaluated. Besides, meat quality during aquaculture should be seriously considered. This result could potentially serve as microbiome indicators in different aquaculture environments. Our study highlights the necessity to incorporate microbiome toxicology research in the aquaculture industry.

\section{Declarations}

Availability of data and materials All data generated or analyzed during this study are included in this published article (and its supplementary information files).

Authors' contributions Jia Du conceived and designed the experiments. Qinhua Liu supported the organization of the project and performed the experiments. Keqin Zhang, Chengxiang Zhang and Gaohua Yao analyzed the data. All contributing authors have read and approved the final version of the manuscript.

Ethics approval Not applicable

Consent to participate Not applicable

Consent of publication Not applicable

Competing interests The authors declare that they have no conflict of interest.

Funding information This work was financially supported by China Postdoctoral Program (2020M270228) and Suzhou Science and Technology Grant (SNG 2018096) in China. 
Acknowledgements We would like to thank Mr. Han Meng and Ms. Naomi Sudo for their assistance in sampling the fish and analyzing the data. We would also like to express our appreciation for all supports from the RD Center of Suzhou Ecoman Bioagriculture Technology, LTD, Suzhou. JS, China.

\section{References}

1. Addo F.G., Zhang S.H., Manirakiza B., Ohore O.E., Shudong Y., 2021, The impacts of straw substrate on biofloc formation, bacterial community and nutrient removal in shrimp ponds. Bioresource Technol. 326, 124727.

2. Beale D.J, Karpe A.V., Ahmed W., 2016, Beyond Metabolomics: A Review of Multi Omics-Based Approaches. Microb Metabolomics. 289-312.

3. Bjrnevik M., Hansen H., Roth B., Foss A., Vikingstad E., Solberg C., Imsland A.K., 2017, Effects of starvation, subsequent feeding and photoperiod on flesh quality in farmed cod (Gadus morhua). Aquacult. Nutr. 23(2), 285-292.

4. Bolger A.M., Lohse M., Usadel B., 2014, Trimmomatic: a flexible trimmer for Illumina sequence data. Bioinformatics 30, 21142120.

5. Bunnell D.B., Marschall E.A., 2003, Optimal energy allocation to ovaries after spawning. Evol. Ecol. Res. 5, 439-457.

6. Dai L.L., Liu C.Q., Peng L., Song C.F., Li X.L., Tao L., Li G., 2021, Different distribution patterns of microorganisms between aquaculture pond sediment and water. J. Microbiol. 59, 376-388.

7. FAO. 2020. The State of World Fisheries and Aquaculture 2020. Sustainability in action. Rome. https://doi.org/10.4060/ca9229en.

8. Gregory C.J., Kuczynski J., Stombaugh J., Bittinger K.『Bushman F.D.『Costello E.K.『Fierer N.『Peña A.G.『Goodrich J.K.『Gordon J.I., 2010, QIIME allows analysis of high-throughput community sequencing data. Nat. Methods. 7, 335-6.

9. Grujcic V., Nuy J.K., Salcher M.M., Shabarova T., Kasalicky V., Boenigk J., Jensen M., Simek K., 2018, Cryptophyta as major bacterivores in freshwater summer plankton. ISME J. 12, 1668-1681.

10. Kanehisa M., Araki M., Goto S., Hattori M., Hirakawa M., Itoh M., Katayama T., Kawashima S., Okuda S., Tokimatsu T., 2008, KEGG for linking genomes to life and the environment. Nucleic Acids Res. 36, 480-484.

11. Kim D., Langmead B., Salzberg S.L., 2015, HISAT: a fast spliced aligner with low memory requirements. Nat. Methods, 12, 357U121.

12. Kumar A., Ng D.H.P., Wu Y.C., Cao B.M., 2019, Microbial community composition and putative biogeochemical functions in the sediment and water of tropical granite quarry lakes. Microb. Ecol. 77, 1-11.

13. Liu Q., Jia Y., Gao Y., Wang A., Goudie C., 2006, Biological characteristics and intensive-culture management of American shad. Fish. Mod. 1, 26-27.

14. Liu Q.H., Zheng Y.H., Fu L., Simco B.A., Goudie C.A., 2021, Brood-stock management and natural spawning of American shad (Alosa sapidissima) in a recirculating aquaculture system. Aquaculture 532,735952.

15. Lobo. 2008, Basic Local Alignment Search Tool (BLAST). J Mol. Biol., 215(3), 403-410.

16. Mahmoudi N., Beaupré S.R., Steen A.D., Pearson, A., 2017, Sequential bioavailability of sedimentary organic matter to heterotrophic bacteria. Environ. Microbiol. 19, 2629-2644.

17. Masrizal U.Z., Zein M, et al. 2015, Effect of energy, lipid and protein content in broodstock diets on spawning fecundity and eggs quality of giant gourami (Ospheronemus gouramy Lac). Pakistan J. Nutr. 14, 412-416.

18. Nack C.C., Swaney D.P., Limburg K.E., 2019, Historical and projected changes in spawning Phenologies of American Shad and Striped bass in the Hudson River Estuary. Mar. Coast. Fish. 11, 271-284.

19. National Bureau of Fisheries and China Society of Fisheries. 2019. China Fisheries Statistical Yearbook 2019. China Agricultural Press, Beijing, P. R. China.

20. Ostbye T.K.K., Ruyter B., Standal I. B., Stien L.H., Bahuaud D., Dessen J.E., Mørkøre T., 2018, Functional amino acids stimulate muscle development and improve fillet texture of Atlantic salmon. Aquacult. Nutr. 24(1), 14-26.

21. Qiu W.H., Liu X.J., Yang F., Li R.Z., Xiong Y., Fu C.X., Li G.R., Liu S., Zheng C.M., Liu X., 2020, Single and joint toxic effects of four antibiotics on some metabolic pathways of zebrafish (Danio rerio) larvae, Sci. Total. Environ. 716, 137062. 
22. Reyon D., Tsai S.Q., Khayter C., Foden J.A., Sander J.D., Joung J.K., 2012, FLASH assembly of TALENs for high-throughput genome editing. Nat. Biotechnol. 30, 460-5.

23. Rognes T., Flouri T., Nichols B., Quince C., Mahe F., 2016, VSEARCH: a versatile open source tool for metagenomics. Peer J. 4, e2584.

24. Shaughnessy A.R., Sloan J.J., Corcoran M.J., Hasenmueller E.A., 2019, Sediments in agricultural reservoirs act as sinks and sources for nutrients over various timescales. Water Resour. Res. 55, 5985-6000.

25. St. Pierre, R., 1992. Restoration of American Shad to the Susquehanna River, Annual Progress Report, 1991. Susquehanna River Anadromous Fish Restoration Committee, Harrisburg, Pennsylvania, USA.

26. Su J.Z., Mei L.Y., Xi L.W., Gong Y.L., Yang Y.X., Jin J.Y., Liu H.K., Zhu X.M., Xie S.Q., Han D., 2021, Responses of glycolysis, glycogen accumulation and glucose-induced lipogenesis in grass carp and Chinese longsnout catfish fed high-carbohydrate diet. Aquaculture 533, 736146.

27. Sun L.J., Cao X.Y., Tan C.Y., Deng Y.Q., Cai R.Z., Peng X., Bai J., 2020, Analysis of the effect of cadmium stress on root exudates of Sedum plumbizincicola based on metabolomics. Ecotox. Envrion. Safe. 205, 111152.

28. Ul-Hasan S., Bowers R.M., Figueroa-Montiel A., Licea-Navarro A.F., Beman J.M., Woyke T., Nobile, C.J., 2019, Community ecology across bacteria, archaea and microbial eukaryotes in the sediment and seawater of coastal Puerto Nuevo, Baja California. PLoS One 14, e0212355.

29. Wang Q., Garrity G.M., Tiedje J.M., Cole J.R., 2007, Naive Bayesian classifier for rapid assignment of rRNA sequences into the new bacterial taxonomy. Appl. Environ. Microbiol. 73, 5261-7.

30. Wörmer L, Hoshino T, Bowles M.W, Viehweger B., Adhikari R.R., Xiao N., Uramoto G., Könneke M., Lazar C.S., Morono Y., 2019, Microbial dormancy in the marine subsurface: Global endospore abundance and response to burial. Sci. Adv. 5, eaav1024.

31. Xu Z.X., Li T.Y., Li E.C., Chen K., Ding Z.L., Qin J.G., Chen L.Q., Ye J.Y., 2016, Comparative transcriptome analysis reveals molecular strategies of oriental river prawn Macrobrachium nipponense in response to acute and chronic nitrite stress. Fish Shellfish Immun. 48, 254-265.

32. Yang H.J. , Zhang Y., Shi H.Z., 2020, Analysis of genes and metabolites associated with propamocarb hydrochloride response in tobacco. Agron. J. 112, 4939-4950.

33. Ye J., Liu X., Wang Z., Wang K., 2011, Effect of partial fish meal replacement by soybean meal on the growth performance and biochemical indices of juvenile Japanese flounder Paralichthys olivaceus. Aquacult Int 19, 143-153.

34. Zhao X.Y., Wei Z., Zhao Y., Xi B., Wang X., Zhao T., Zhang X., Wei Y., 2015, Environmental factors influencing the distribution of ammonifying and denitrifying bacteria and water qualities in 10 lakes and reservoirs of the Northeast, China. Microb. Biotechnol. 8, 541-548.

35. Zhou L.F., Zhao B.W., Guan N.N., Wang W.M., Gao Z.X., 2017, Plasma metabolomics profiling for fish maturation in blunt snout bream. Metabolomics 13, 40.

\section{Tables}

Table 1

Biological processes and molecular functions of the most differentially expressed genes (top-twenty) after culture different cultured environments. 


\begin{tabular}{|c|c|c|c|c|c|c|}
\hline Gene id & $\begin{array}{l}\text { Gene } \\
\text { name }\end{array}$ & Gene description & Base Mean & pValue & Fold Change & $\begin{array}{l}\text { Molecular } \\
\text { Function }\end{array}$ \\
\hline \multicolumn{7}{|l|}{ Up-regulated } \\
\hline As04G004894 & Rnh1 & Ribonuclease inhibitor & 15.45035579 & 0.00364084 & 102.2612438 & protein binding \\
\hline As23G026860 & SDHC & $\begin{array}{l}\text { Succinate dehydrogenase } \\
\text { cytochrome b560 subunit }\end{array}$ & 78.17066854 & $5.82 \mathrm{E}-16$ & 61.00085809 & $\begin{array}{l}\text { succinate } \\
\text { dehydrogenase } \\
\text { activity }\end{array}$ \\
\hline As07G008885 & mogat2-a & $\begin{array}{l}\text { 2-acylglycerol 0- } \\
\text { acyltransferase 2-A }\end{array}$ & 7.283084764 & 0.000139609 & 43.74880818 & $\begin{array}{l}\text { transferase } \\
\text { activity, } \\
\text { transferring } \\
\text { acyl groups } \\
\text { other than } \\
\text { amino-acyl } \\
\text { groups }\end{array}$ \\
\hline As11G013878 & PGC & Gastricsin & 173251.9722 & 0.046261289 & 38.22168004 & $\begin{array}{l}\text { aspartic-type } \\
\text { endopeptidase } \\
\text { activity }\end{array}$ \\
\hline As04G004598 & Unknown & Cystatin & 30708.22391 & 0.042029946 & 34.39259952 & $\begin{array}{l}\text { cysteine-type } \\
\text { endopeptidase } \\
\text { inhibitor } \\
\text { activity }\end{array}$ \\
\hline As20G023043 & SBF1 & $\begin{array}{l}\text { Myotubularin-related } \\
\text { protein } 5\end{array}$ & 7.503540635 & 0.017474453 & 22.05166777 & $\begin{array}{l}\text { phosphatase } \\
\text { regulator } \\
\text { activity }\end{array}$ \\
\hline As08G010278 & cyp1a1 & Cytochrome P450 1A1 & 12.52643903 & 0.001242407 & 19.99463312 & $\begin{array}{l}\text { iron ion } \\
\text { binding }\end{array}$ \\
\hline As21G023933 & UMAD1 & $\begin{array}{l}\text { UBAP1-MVB12-associated } \\
\text { (UMA)-domain containing } \\
\text { protein } 1\end{array}$ & 3.200192741 & 0.035681065 & 18.66265885 & \\
\hline As15G019128 & timm23 & $\begin{array}{l}\text { Mitochondrial import inner } \\
\text { membrane translocase } \\
\text { subunit Tim23 }\end{array}$ & 26.86638894 & 1.75E-09 & 18.64867257 & $\begin{array}{l}\text { TIM23 } \\
\text { mitochondrial } \\
\text { import inner } \\
\text { membrane } \\
\text { translocase } \\
\text { complex }\end{array}$ \\
\hline As02G001904 & Unknown & $\begin{array}{l}\text { L-rhamnose-binding lectin } \\
\text { CSL3 }\end{array}$ & 93.35504268 & 0.028271521 & 17.97440459 & $\begin{array}{l}\text { carbohydrate } \\
\text { binding }\end{array}$ \\
\hline \multicolumn{7}{|l|}{$\begin{array}{l}\text { Down- } \\
\text { regulated }\end{array}$} \\
\hline As24G026970 & ATP2A1 & $\begin{array}{l}\text { Sarcoplasmic/endoplasmic } \\
\text { reticulum calcium ATPase } \\
1\end{array}$ & 95.17048306 & 0.034741487 & 0.496868494 & $\begin{array}{l}\text { nucleotide } \\
\text { binding }\end{array}$ \\
\hline As15G018679 & Unknown & Unknown & 170.1634723 & 0.005104726 & 0.496303833 & Unknown \\
\hline As13G016569 & SSH1 & $\begin{array}{l}\text { Protein phosphatase } \\
\text { Slingshot homolog } 1\end{array}$ & 34.07582779 & 0.047482077 & 0.493473838 & $\begin{array}{l}\text { protein } \\
\text { tyrosine } \\
\text { phosphatase } \\
\text { activity }\end{array}$ \\
\hline As14G017421 & Dusp8 & $\begin{array}{l}\text { Dual specificity protein } \\
\text { phosphatase } 8\end{array}$ & 146.4822989 & 0.032029515 & 0.49267269 & $\begin{array}{l}\text { protein } \\
\text { tyrosine } \\
\text { phosphatase } \\
\text { activity }\end{array}$ \\
\hline As02G001872 & PLA2G1B & Phospholipase A2 & 3692.414228 & 0.001021302 & 0.491257297 & phospholipase \\
\hline
\end{tabular}




\begin{tabular}{|lllllll|}
\hline As08G010690 & SLC4A4 & $\begin{array}{l}\text { Electrogenic sodium } \\
\text { bicarbonate cotransporter 1 }\end{array}$ & 49.49409386 & 0.021895553 & 0.490846315 & $\begin{array}{l}\text { A2 activity } \\
\text { inorganic } \\
\text { anion } \\
\text { exchanger } \\
\text { activity }\end{array}$ \\
\hline As13G016353 & SLC27A4 & $\begin{array}{l}\text { Long-chain fatty acid } \\
\text { transport protein 4 }\end{array}$ & 37.70459613 & 0.034515932 & 0.486819323 & $\begin{array}{l}\text { catalytic } \\
\text { activity }\end{array}$ \\
\hline As19G022184 & Tnni1 & $\begin{array}{l}\text { Troponin I, slow skeletal } \\
\text { muscle }\end{array}$ & 3809.149612 & 0.033993754 & 0.486635234 & $\begin{array}{l}\text { troponin } \\
\text { complex }\end{array}$ \\
\hline As16G019505 & Myh7 & Myosin-7 & 2666.635533 & 0.027133197 & 0.485618664 & motor activity \\
\hline As22G025262 & PCDHA8 & Protocadherin alpha-8 & 74.02863914 & 0.032156152 & 0.483550399 & $\begin{array}{l}\text { calcium ion } \\
\text { binding }\end{array}$ \\
\hline
\end{tabular}

Table 2

Biological processes and molecular functions of the commom differentially expressed genes

\begin{tabular}{|c|c|c|c|c|}
\hline Gene id & $\begin{array}{l}\text { Gene } \\
\text { name }\end{array}$ & Gene description & Regulation & Molecular Function \\
\hline As13G016035 & Unkown & Phospholipase A2 GL16-1 & $\begin{array}{l}\text { Down- } \\
\text { regulated }\end{array}$ & $\begin{array}{l}\text { glycerophospholipid } \\
\text { metabolism }\end{array}$ \\
\hline As21G024434 & Ipcat1 & Lysophosphatidylcholine acyltransferase 1 & $\begin{array}{l}\text { Up- } \\
\text { regulated }\end{array}$ & calcium ion binding \\
\hline As04G005193 & Pla2g4c & Cytosolic phospholipase A2 gamma & $\begin{array}{l}\text { Up- } \\
\text { regulated }\end{array}$ & phospholipase activity \\
\hline As04G005148 & PNPLA6 & Cytosolic phospholipase A2 gamma & $\begin{array}{l}\text { Up- } \\
\text { regulated }\end{array}$ & Unkown \\
\hline As02G001872 & PLA2G1B & Phospholipase A2 & $\begin{array}{l}\text { Down- } \\
\text { regulated }\end{array}$ & phospholipase $\mathrm{A} 2$ activity \\
\hline As07G009244 & SAT2 & Diamine acetyltransferase 2 & $\begin{array}{l}\text { Down- } \\
\text { regulated }\end{array}$ & pN-acetyltransferase activity \\
\hline As15G018635 & PDE10A & $\begin{array}{l}\text { cAMP and cAMP-inhibited cGMP 3',5'-cyclic } \\
\text { phosphodiesterase } 10 \mathrm{~A}\end{array}$ & $\begin{array}{l}\text { Down- } \\
\text { regulated }\end{array}$ & $\begin{array}{l}\text { 3',5'-cyclic-nucleotide } \\
\text { phosphodiesterase activity }\end{array}$ \\
\hline As13G016748 & HMGCS1 & $\begin{array}{l}\text { Hydroxymethylglutaryl-CoA synthase, } \\
\text { cytoplasmic }\end{array}$ & $\begin{array}{l}\text { Up- } \\
\text { regulated }\end{array}$ & catalytic activity \\
\hline As23G026314 & TDH & L-threonine 3-dehydrogenase, mitochondrial & $\begin{array}{l}\text { Down- } \\
\text { regulated }\end{array}$ & catalytic activity \\
\hline
\end{tabular}


Figures

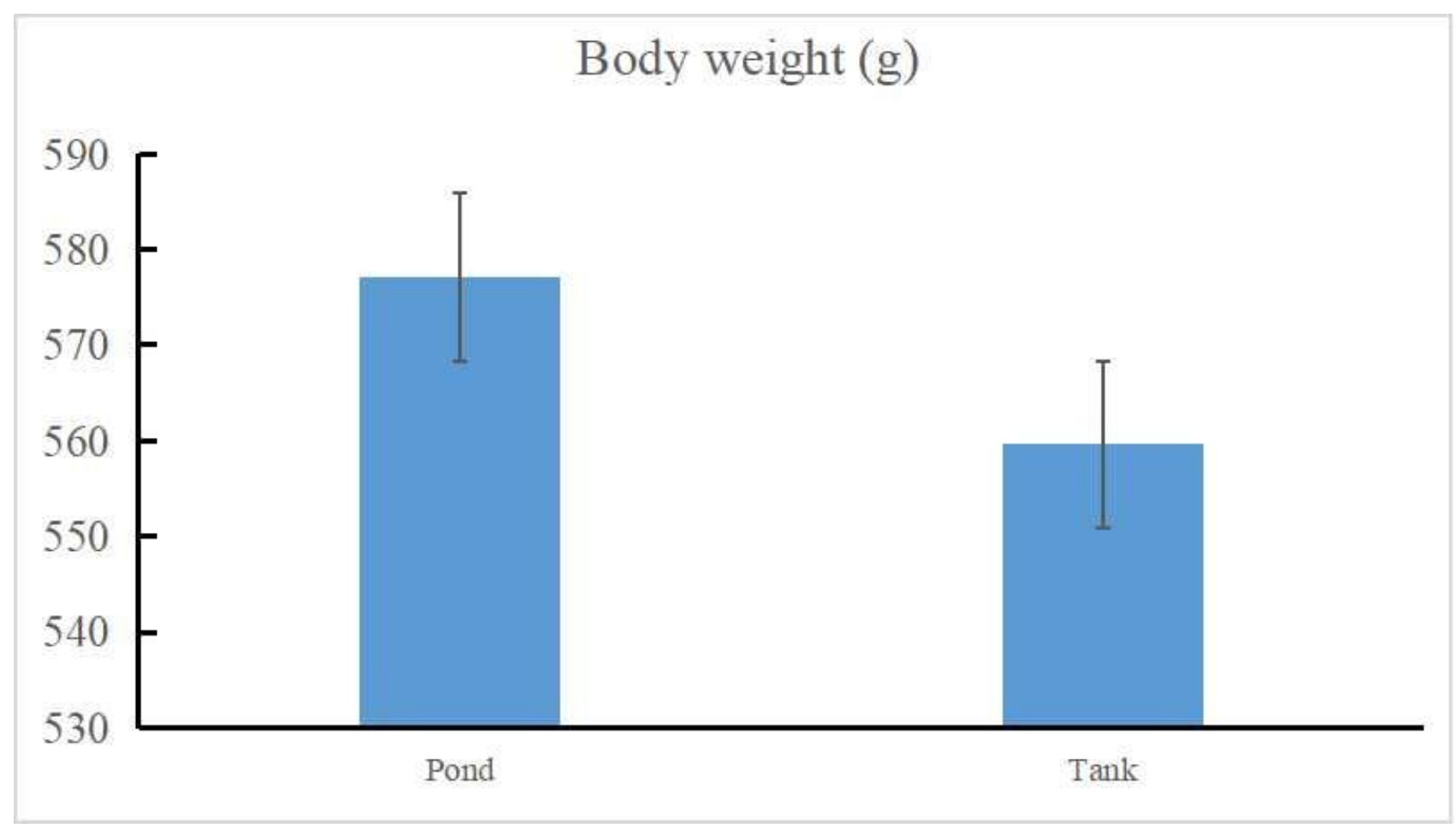

Figure 1

Body weight in tank aquaculture group and pond aquaculture group. 
A

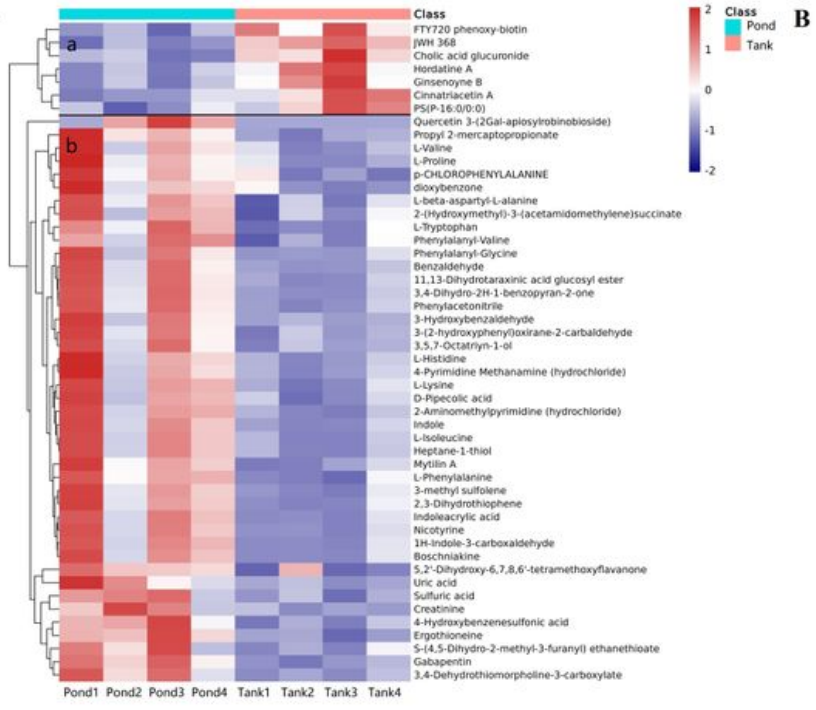

C

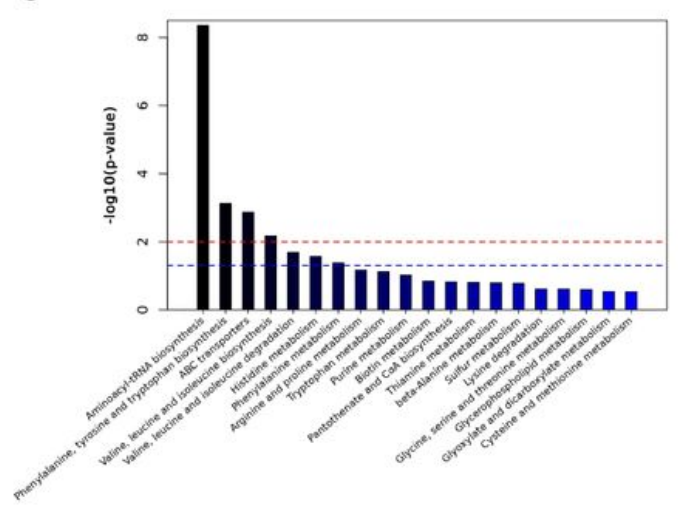

D
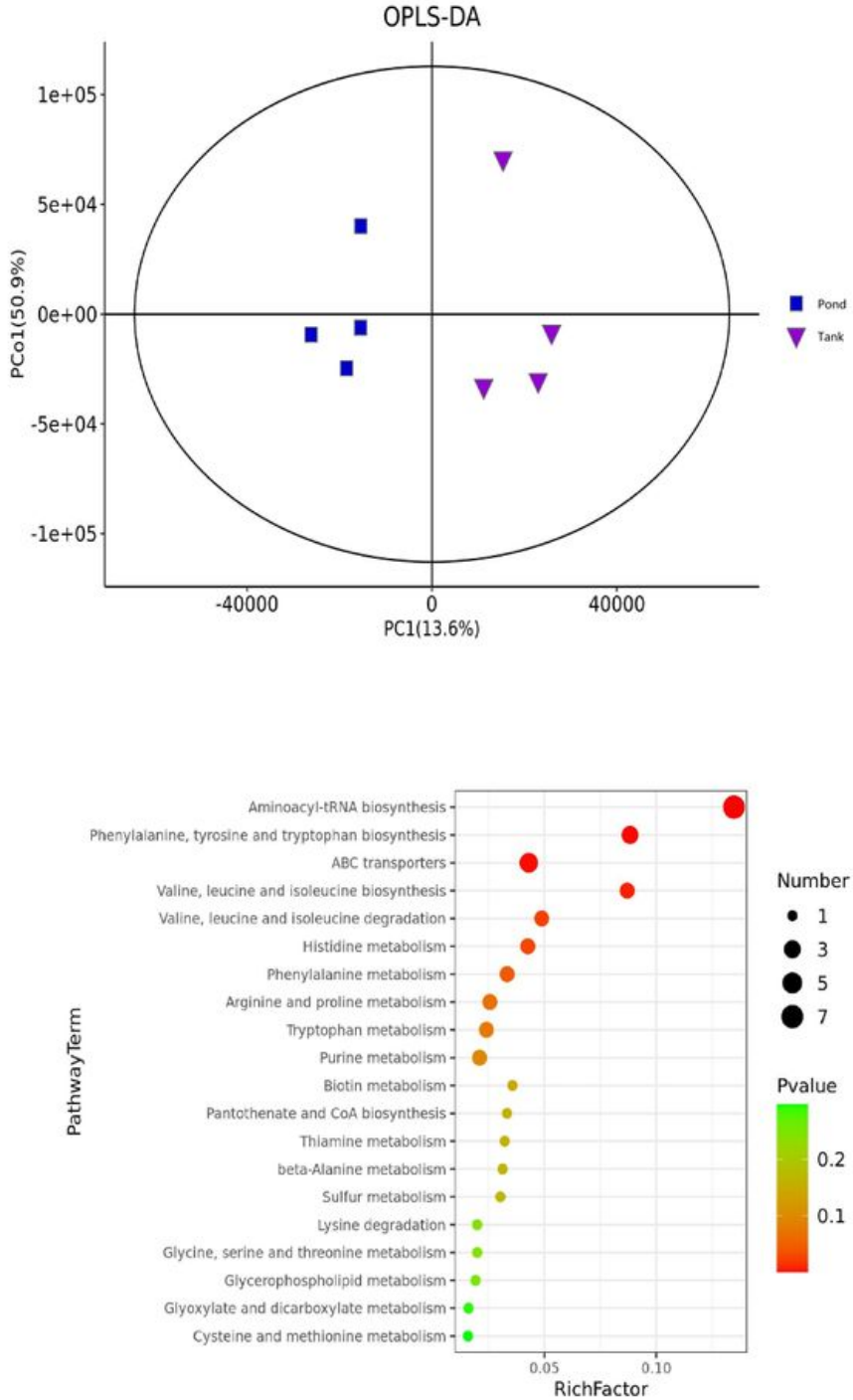

Figure 2

(A) Heatmap of the statistically significant tentatively identified metabolites whose concentrations changed between tank aquaculture group and pond aquaculture group, (a) down-regulated metabolites; (b) up-regulated metabolites; (B) OPLS-DA score plot shows clear separation of metabolic profiles between the two groups; (C) Top-20 metabolic pathways enrichment map (the red line indicates $P=0.01$, and the blue line indicates $P=0.05$ ) (D) Bubble diagram of metabolic pathways, the ordinate indicates the name of metabolic pathways and the abscissa indicates the enrichment factors (Rich factor, Rich factor=significantly different number of metabolites/total number of metabolites in the pathway). 
A

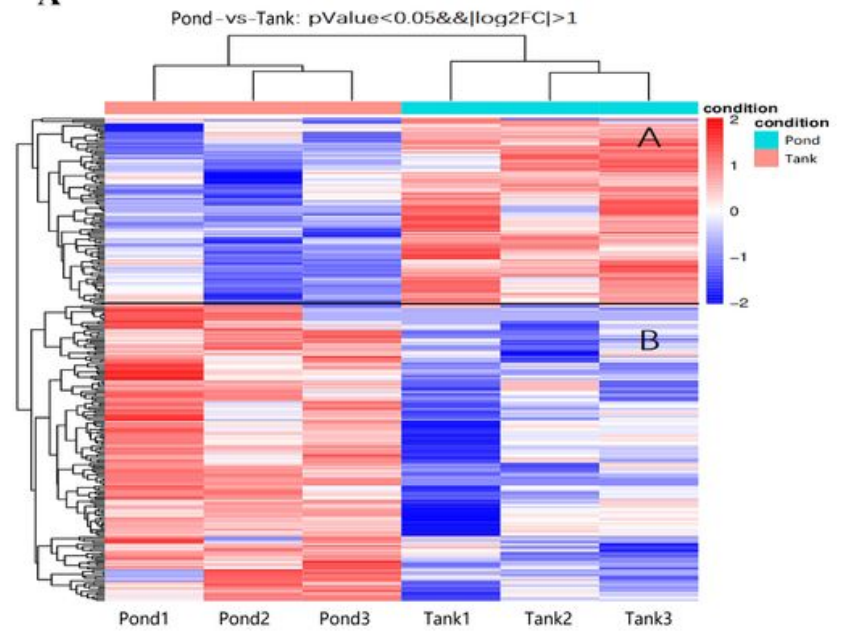

C

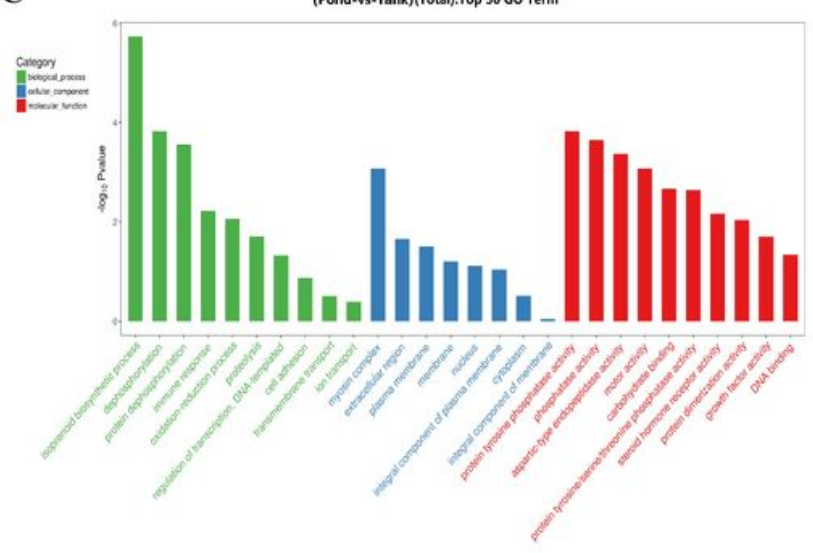

B

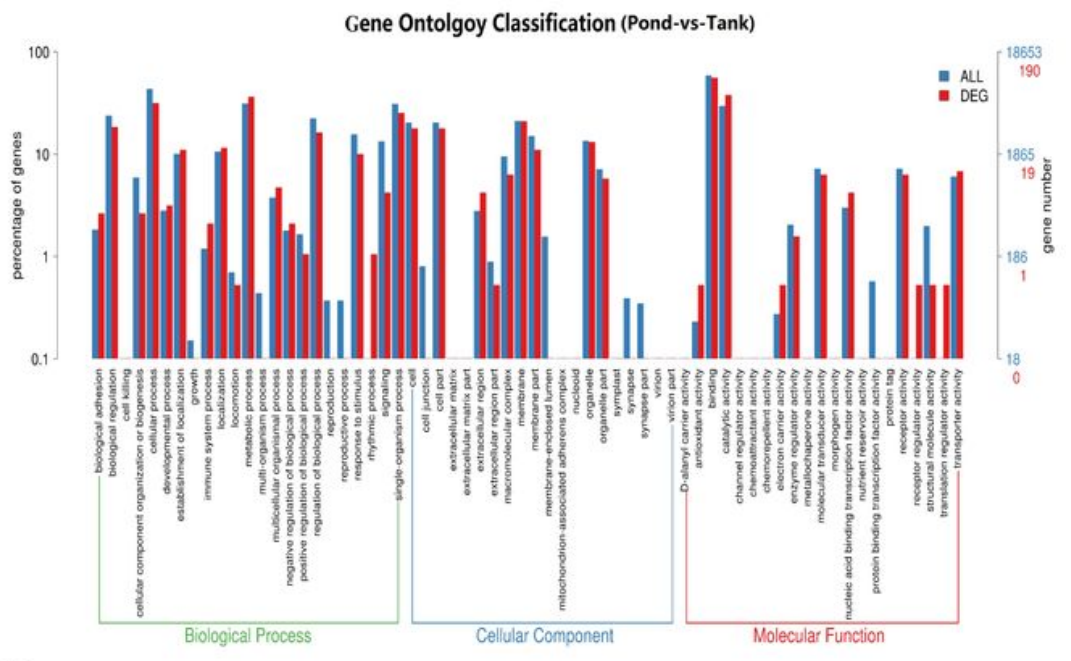

D

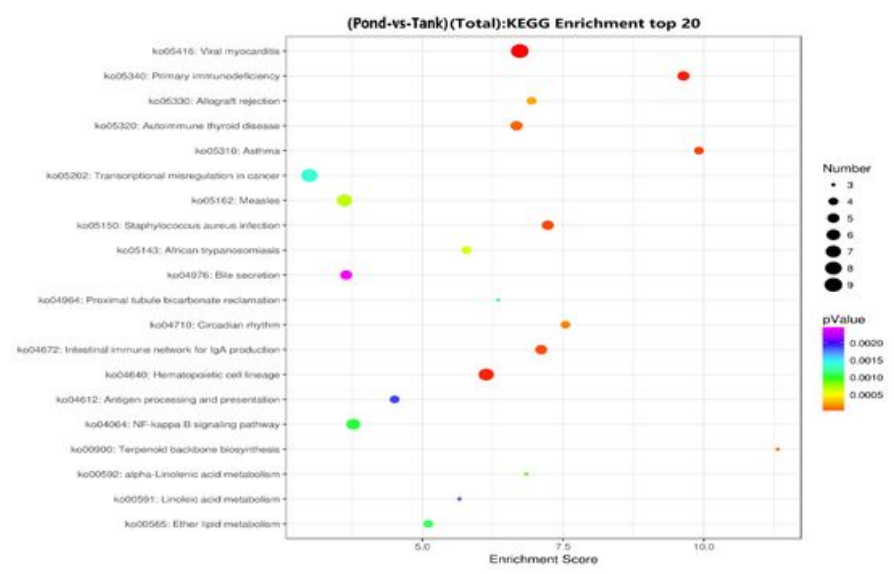

Figure 3

(A) Heatmap of the DEGs in two groups; (B) The distribution comparison diagram of DEGs and all genes by GO enrichment analysis at level 2; (C) GO enrichment analysis: the horizontal axis represents Go entry name and the vertical axis represents $\log 10$ (p value); (D) Bubble diagram of KEGG enrichment analysis TOP-20 of DEGs.

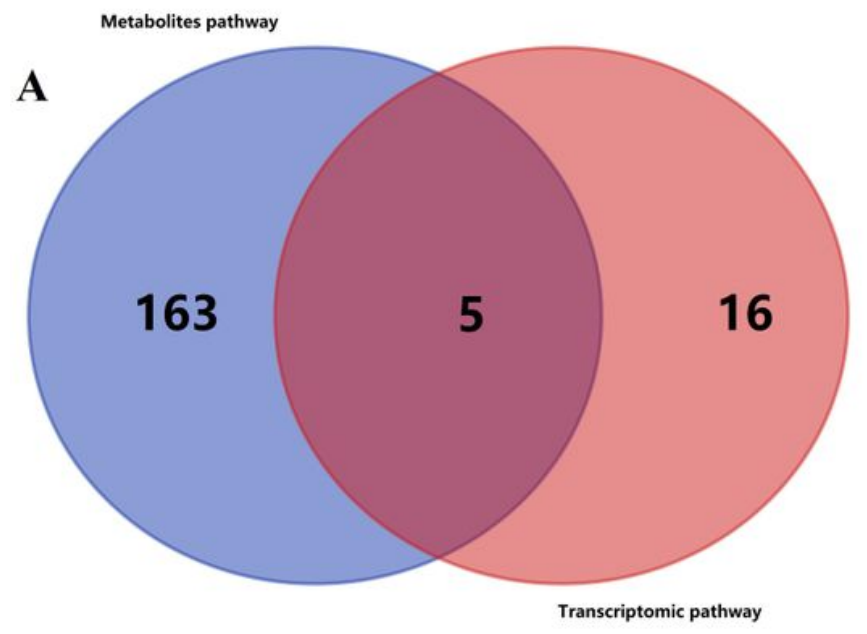

B

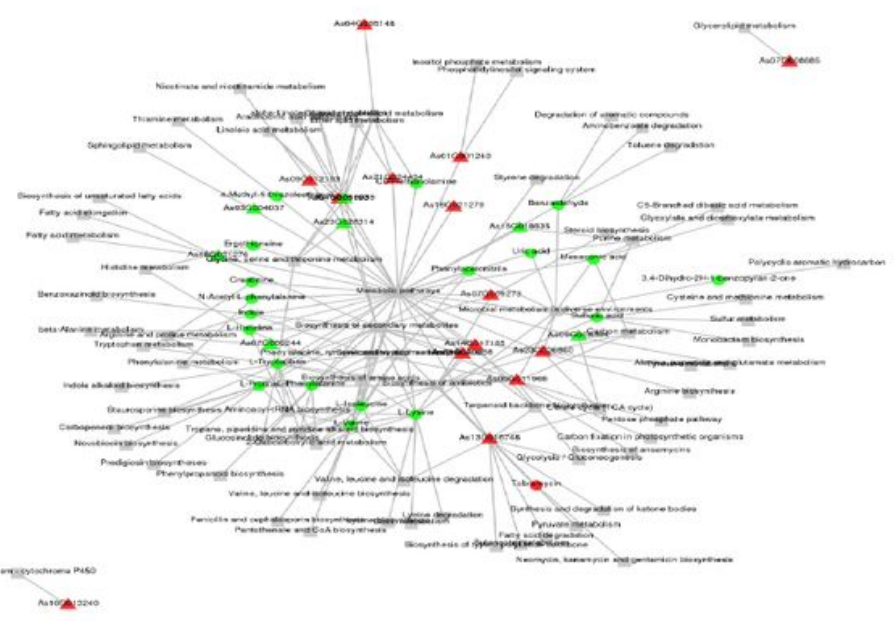

type - Metabolites $\Delta$ gene $\mathbf{m}$ map 
(A) Venn diagrams of the common KEGG pathways (Blue represents metabolites pathways and red represents transcriptomic pathways) (B) The association network map of metabolites (represented by their KEGG C-codes) and genes (represented by their gene ID).

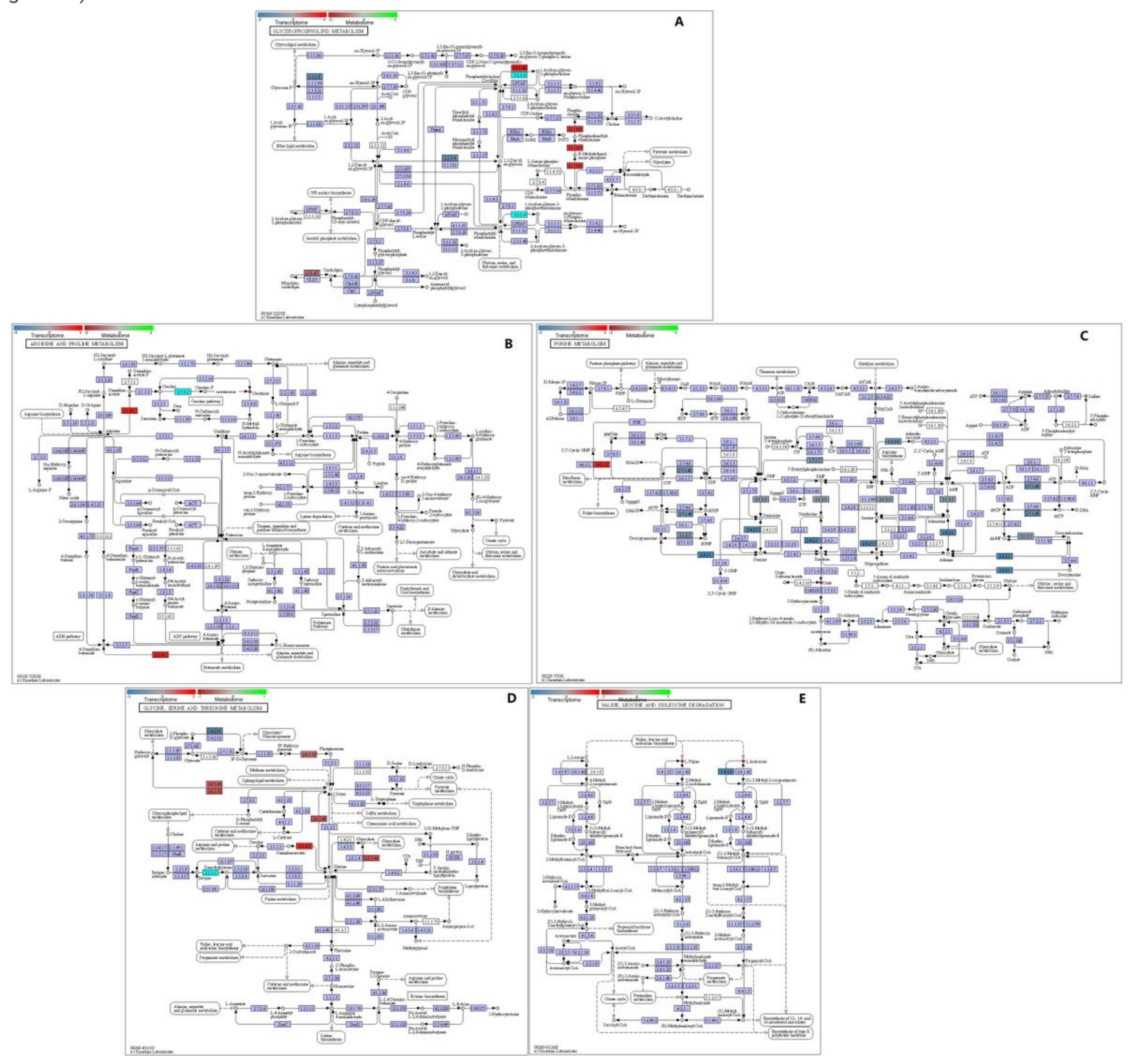

Figure 5

Metabolic KEGG maps (A) Glycerophospholipid metabolism; (B) Arginine and proline metabolism; (C) Purine metabolism; (D) Glycine, serine and threonine metabolism; (E) Valine, leucine and isoleucine degradation; the boxes are genes, the circles are metabolites, the red is up-regulated, the green is down-regulated, and the yellow is both up-regulated and down-regulated. 
A
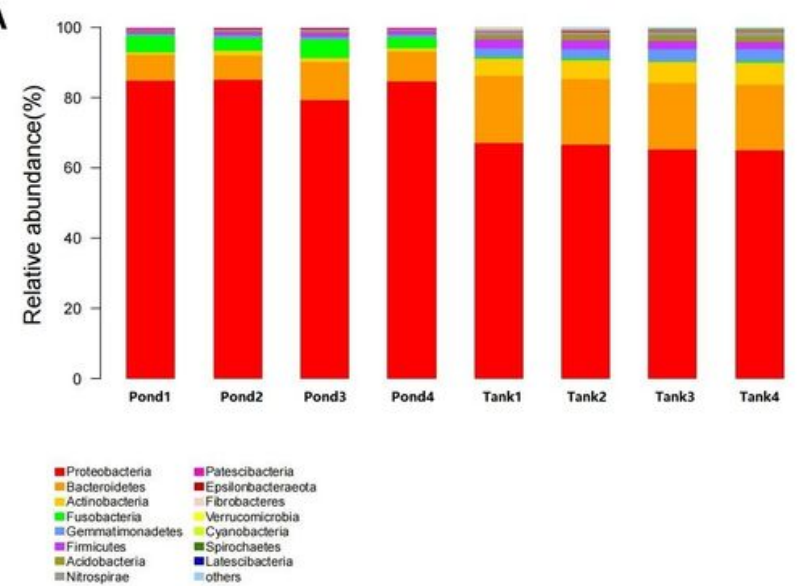

C

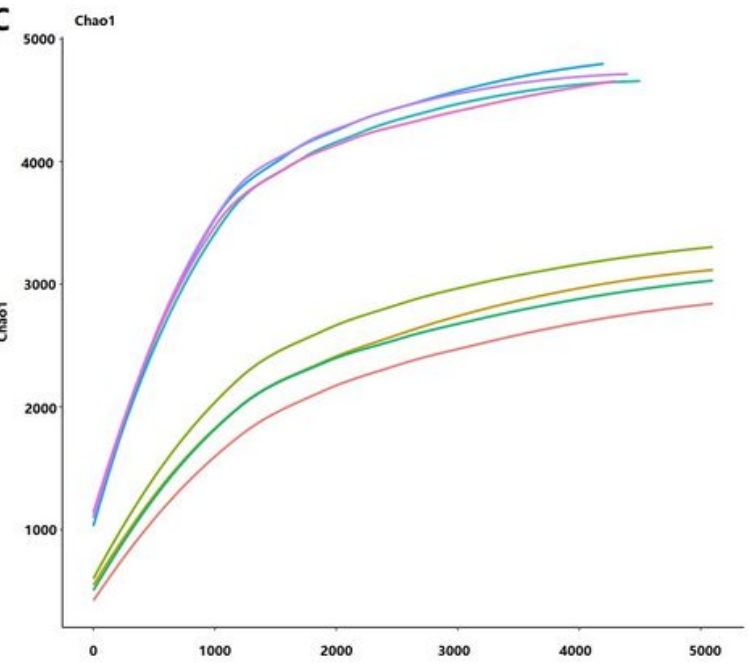

B

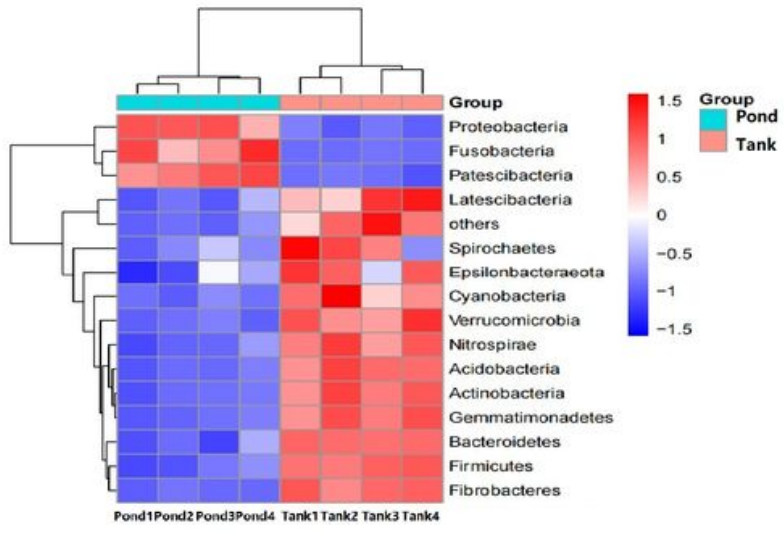

D

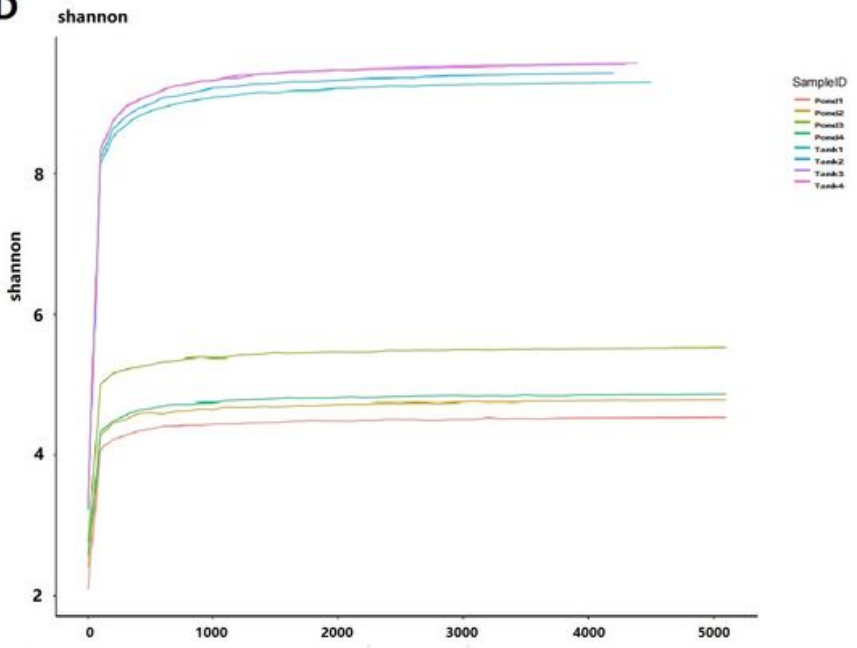

Figure 6

(A) Microbiome composition at the phylum level samples from tank aquaculture group and pond aquaculture group. The top fifteen phyla were reported for each sample, and all other phyla were grouped into "Other" ; (B) The cluster tree of two groups. Left represents the clustering of species; Above cluster branch Group represents samples from different groups; Orange indicates relatively high species abundance and blue indicates relatively low species abundance; Chao 1 (C) and Shannon (D) indexes of sediment samples in $\mathrm{GH}$ and WH groups. 

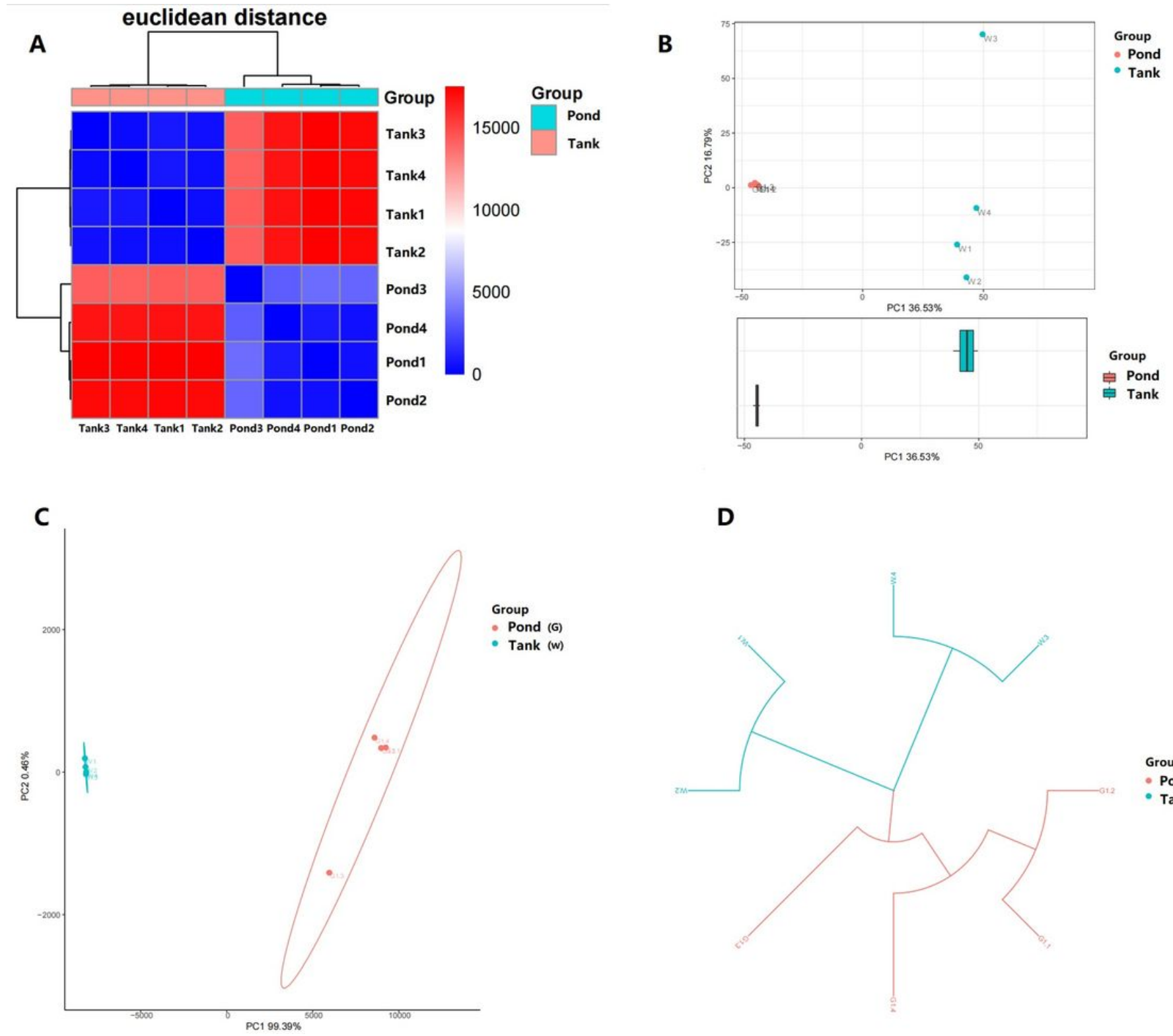

D

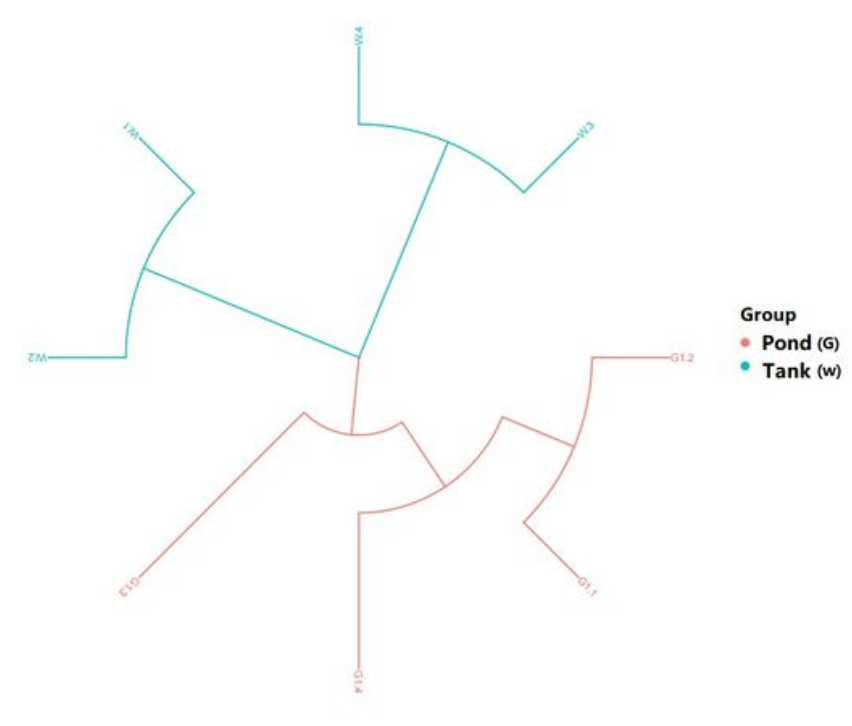

Figure 7

(A) Beta diversity index; (B) PCA analysis; (C) PCoA analysis; (D) UPGMA sample hierarchical cluster analysis. 

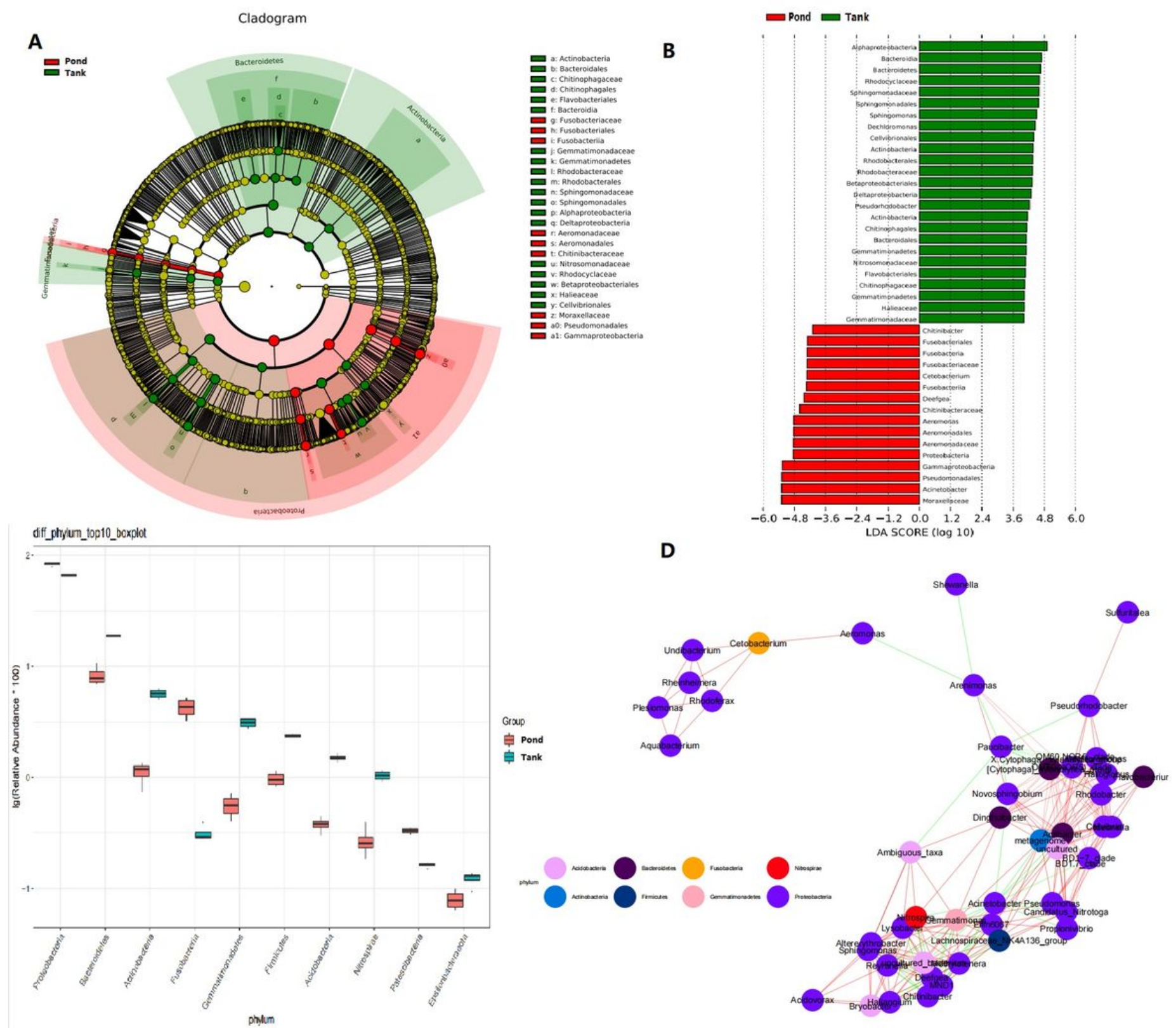

Figure 8

(A) LEfSe analysis of microbial abundance, the cladogram of microbial communities; (B) LDA score identifified the size of differentiation between $\mathrm{GH}$ and WH groups. Data presented as the mean \pm SEM of three replicates and an asterisk indicates the statistical signifificance ( $\mathrm{p}$-value < 0.05 ) between TET treated and control groups; $(\mathrm{C})$ The relative abundunce of top-ten phylum in two groups; (D) The species correlation network map at phylum levels.

\section{Supplementary Files}

This is a list of supplementary files associated with this preprint. Click to download.

- Supplementaryfigure.1.jpg

- Supplementaryfigure.2.jpg

- Supplementaryfigure.3.jpg

- Supplementarymaterial1.xlsx

- Supplementarymaterial2.xlsx 